\title{
CDISC SEND Subject Characteristic Test Name Terminology
}

National Cancer Institute

\section{Source}

National Cancer Institute. CDISC SEND Subject Characteristic Test Name Terminology. NCI Thesaurus. Code C89980.

The terminology that includes concepts relevant to the Clinical Data Interchange Standards Consortium (CDISC) Standard for the Exchange of Non-clinical Data (SEND) subject characteristics test names. 